Pacific

Journal of

Mathematics

A SPLITTING THEOREM FOR ALEXANDROV SPACES

YUKIHIRO MASHIKO

Volume $204 \quad$ No. 2

June 2002 


\title{
A SPLITTING THEOREM FOR ALEXANDROV SPACES
}

\author{
YUKIHIRO MASHIKO
}

\begin{abstract}
We use a notion of differentiability for functions on Alexandrov spaces and prove a splitting theorem for Alexandrov spaces admitting affine functions with such differentiability.
\end{abstract}

\section{Introduction.}

A classical result of Toponogov [12] states that if a complete Riemannian manifold $M$ with nonnegative sectional curvature contains a straight line, then $M$ is isometric to the metric product of a nonnegatively curved manifold and a line. We then know that the Busemann function associated with the straight line is an affine function, namely, a function which is affine on each unit speed geodesic in the one variable sense. After the theorem, many generalizations were proved. Cheeger-Gromoll's theorem [2] is the most excellent one among them.

An Alexandrov space with curvature bounded below by $\kappa \in R$ is a locally compact, complete and path connected inner metric space on which the triangle comparison theorem holds (see [1]). For simplicity, we denote by curv $\geq \kappa$ the lower curvature bound. The direct generalization of the Toponogov theorem for Alexandrov spaces with curv $\geq 0$ was proved early in 1967 by A. Milka [8]. We see that this is essentially implied by the rigidity of geodesic triangles and hinges in the Global Comparison Theorem (see Fact 1.0).

The author has shown in [7] that if a 2-dimensional Alexandrov space $X$ with curv $\geq-\kappa^{2}$ without boundary admits a nontrivial affine function, then $X$ is isometric to flat $R^{2}$ or flat $S^{1} \times R$. In the present paper, we extend this to higher dimensional Alexandrov spaces, possibly with nonempty boundary, admitting affine functions with a new notion of differentiability. Innami [6] showed that every complete Riemannian manifold admitting a nontrivial affine function splits isometrically into the metric product of a line and a Riemannian manifold. Affine functions on complete Riemannian manifolds naturally possess the differentiability introduced in this paper.

We shall define some notion needed to state our main theorem. Let $X$ be an $n$-dimensional Alexandrov space with curv $\geq-\kappa^{2}$ and $n \geq 2, \kappa>0$. We denote by $p q$ a minimal geodesic from $p$ to $q$ and by $|p, q|$ the distance 
between $p$ and $q$. We put

$$
\widetilde{\Sigma}_{p} X:=\{p q \mid q \in X-\{p\}\} / \sim,
$$

where the equivalence relation $\sim$ is defined such that $p q \sim p r$ iff $p q \subset p r$ or $p q \supset p r$. Then the space of directions $\Sigma_{p} X$ at $p$ in $X$ is defined to be the metric completion of $\widetilde{\Sigma}_{p} X$. For $u \in \Sigma_{p} X$ we denote by $\gamma_{u}$ the geodesic tangent to $u$ with $\gamma_{u}(0)=p$ if it exists. For a function $f: X \longrightarrow R$, we define the directional derivative $\widetilde{d}_{p} f: \widetilde{\Sigma}_{p} X \longrightarrow R$ at $p$ by $\widetilde{d}_{p} f(u):=(f \circ$ $\left.\gamma_{u}\right)_{+}^{\prime}(0)$ if the right-hand derivative exists. We denote by $d_{p} f: \Sigma_{p} X \longrightarrow R$ the continuous extension of $\widetilde{d}_{p} f$ if it exists. Remarking that $\Sigma_{p} X$ is an Alexandrov space with curv $\geq 1$, we consider the composition $\widetilde{d}_{u_{1}} \circ d_{p}$ of the two operators $\widetilde{d}_{u_{1}}$ and $d_{p}$ for $p \in X$ and $u_{1} \in \Sigma_{p} X$. We put $\widetilde{d} f_{p, u_{1}}:=$ $\widetilde{d}_{u_{1}} \circ d_{p} f$ and denote by $d f_{p, u_{1}}: \Sigma_{u_{1}} \Sigma_{p} X \longrightarrow R$ the cotinuous extension of $\widetilde{d} f_{p, u_{1}}: \widetilde{\Sigma}_{u_{1}} \Sigma_{p} X \longrightarrow R$. Repeating the procedure, we define for $k$ with $1 \leq k<n=\operatorname{dim} X$,

$$
\begin{aligned}
& \Sigma^{k} X:=\left\{\left(p, u_{1}, u_{2}, \ldots, u_{k}\right) \mid p\right. \in X, u_{1} \in \Sigma_{p} X, \\
& u_{i}\left.\in \Sigma_{u_{i-1}} \Sigma_{u_{i-2}} \cdots \Sigma_{u_{1}} \Sigma_{p} X(i=2, \ldots, k)\right\}, \\
& \widetilde{d} f_{p, u_{1}, u_{2}, \ldots, u_{k}}:=\widetilde{d}_{u_{k}} \circ \cdots \circ d_{u_{2}} \circ d_{u_{1}} \circ d_{p} f: \widetilde{\Sigma}_{u_{k}} \Sigma_{u_{k-1}} \cdots \Sigma_{u_{1}} \Sigma_{p} X \longrightarrow R
\end{aligned}
$$
for a function $f: X \longrightarrow R$ and for $\left(p, u_{1}, u_{2}, \ldots, u_{k}\right) \in \Sigma^{k} X$.

Definition 0.1. A function $f: X \longrightarrow R$ belongs to the class $D^{r}(1 \leq r \leq$ $n=\operatorname{dim} X$ ), or simply, $f$ is of $D^{r}$ class iff $\widetilde{d} f_{p, u_{1}, u_{2}, \ldots, u_{k-1}}$ is defined and has the continuous extension

$$
d f_{p, u_{1}, u_{2}, \ldots, u_{k-1}}: \Sigma_{u_{k-1}} \Sigma_{u_{k-2}} \cdots \Sigma_{u_{1}} \Sigma_{p} X \longrightarrow R
$$

for all $\left(p, u_{1}, u_{2}, \ldots, u_{k-1}\right) \in \Sigma^{k-1} X$ and for all $k$ with $1 \leq k \leq r$. We agree that a function $f: X \longrightarrow R$ is of $D^{1}$ class if and only if $\widetilde{d} f_{p}$ has the continuous extension $d f_{p}$.

To control the behavior of the directional derivatives, we introduce a quantity associated with $f: X \longrightarrow R$ as follows:

$$
\Delta_{1} f(p):=\frac{1}{\mathcal{H}^{n-1}\left(\Sigma_{p} X\right)} \int_{\Sigma_{p} X \ni u} d f_{p}(u) d \mathcal{H}^{n-1}(u), \text { for } p \in X,
$$

where $\mathcal{H}^{n-1}$ denotes the $(n-1)$-dimensional Hausdorff measure on $\Sigma_{p} X$. This means the total flow of the gradient of $f$ at the point $p$. If $f$ is a diffenrentiable function on a Riemannian manifold, then $f$ is of $D^{n}$ class and $\Delta_{1} f(p)=0$ at every point $p$. On the other hand, there exists an Alexandrov space on which $\Delta_{1} f(p) \neq 0$ at some singular point $p$ for an affine function $f$ of $D^{3}$ class and the whole space does not split (see Example 1.4). To avoid this case, we need the following definition. 
Definition 0.2. A function $f: X \longrightarrow R$ is of $D^{r, s}$ class, $s \leq r$, iff $f$ is of $D^{r}$ class and

$$
\Delta_{1} d f_{p, u_{1}, u_{2}, \ldots, u_{k-2}}\left(u_{k-1}\right)=0
$$

for all $\left(p, u_{1}, u_{2}, \ldots, u_{k-1}\right) \in \Sigma^{k-1} X$ and for all $k$ with $1 \leq k \leq s$.

With these definitions, we now state our main theorem of this paper:

Theorem A. Let $X$ be an $n$-dimensional Alexandrov space with curv $\geq$ $-\kappa^{2}$. Then, $X$ admits a nontrivial affine function $\varphi: X \longrightarrow R$ of $D^{2,2}$ class if and only if $X$ is isometric to the metric product $\widetilde{X} \times R$, where $\widetilde{X}$ is an $(n-1)$-dimensional Alexandrov space with curv $\geq-\kappa^{2}$.

Since every constant function on $X$ is an affine function of $D^{2,2}$ class, the dimension of the space of all affine functions on $X$ of $D^{2,2}$ class is at least one. Thus we obtain the following corollary:

Corollary B. The linear space of all affine functions on $X$ of $D^{2,2}$ class is of dimension $k+1$ if and only if $X$ is isometric to $\widetilde{X} \times R^{k}$, where $\widetilde{X}$ does not admit any nontrivial affine function of $D^{2,2}$ class.

For the proof of Theorem A, it suffices to show that, for each minimal geodesic $\gamma$ in $X$, there exists a totally geodesic and flat strip including $\gamma$. Under the assumption that $X$ admits an affine function of $D^{2,2}$ class, we will show in Proposition 4.3 that the strip is spanned by the gradient curves of $\varphi$. Recently, G. Perelman and A. Petrunin [10] considered the existence of gradient curves in more general situation. The arguments in this paper is more elementary than theirs, and the author believes that his arguments will be shortend by their existence theorem.

\section{Preliminaries and examples.}

Throughout this paper, let $X$ be an Alexandrov space with curv $\geq-\kappa^{2}$ for $\kappa>0$.

1.0. Global Comparison Theorem. The most basic tool in Alexandrov geometry is the following theorem.

Fact 1.0 (Global Comparison Theorem). (See [1, $\S 3]$, [5, Theorem 1.1] and [4, Appendix].) If $Z$ is an $n$-dimensional Alexandrov space, $n \geq 2$, with curv $\geq k$, then the following holds:

(i) For any triple $\left(p_{0}, p_{1}, p_{2}\right)$ in $Z$ there is a unique (up to isometry) triple $\left(\bar{p}_{0}, \bar{p}_{1}, \bar{p}_{2}\right)$ in $M^{2}(k)$ with $\left|p_{i}, p_{j}\right|=\left|\bar{p}_{i}, \bar{p}_{j}\right|$. Moreover, for any segment $p_{1} p_{2}$ and $0 \leq t \leq\left|p_{1}, p_{2}\right|$

$$
\left|p_{0}, p_{1} p_{2}(t)\right| \geq\left|\bar{p}_{0}, \bar{p}_{1} \bar{p}_{2}(t)\right| \text {. }
$$

(If $k>0$ we must also assume that $\left|p_{0}, p_{1}\right|+\left|p_{1}, p_{2}\right|+\left|p_{2}, p_{0}\right|<2 \pi / \sqrt{k}$.) 
(ii) If equality holds in (a) for some $0<t_{0}<\left|p_{1}, p_{2}\right|$ and $c_{t_{0}}$ is a segment from $p_{0}$ to $p_{1} p_{2}\left(t_{0}\right)$, then $c_{t_{0}}(s), 0<s<\left|p_{0}, p_{1} p_{2}\left(t_{0}\right)\right|$, is joined their limit segments from $p_{0}$ to $p_{1}$ and $p_{2}$, form a surface which has totally geodesic interior and which is isometric to the triangular surface in $M^{2}(k)$ with vertices $\bar{p}_{0}, \bar{p}_{1}, \bar{p}_{2}$.

(iii) For any hinge $\left(p_{0} p_{1}, p_{0} p_{2}\right)$ in $Z$ with $0<\angle\left(p_{0} p_{1}, p_{0} p_{2}\right)<\pi$ we have

$$
\left|p_{1}, p_{2}\right| \leq\left|\bar{p}_{1}, \bar{p}_{2}\right|
$$

where $\left(\bar{p}_{0} \bar{p}_{1}, \bar{p}_{0} \bar{p}_{2}\right)$ is the corresponing hinge in $M^{2}(k)$.

(iv) If equality holds in (b), then $\left(p_{0} p_{1}, p_{0} p_{2}\right)$ spans a surface which has totally geodesic interior and which is isometric to the triangular surface in $M^{2}(k)$ spanned by $\left(\bar{p}_{0} \bar{p}_{1}, \bar{p}_{0} \bar{p}_{2}\right)$. In fact, any such surface is determined uniquely by a segment in $Z$ between interior points of $p_{0} p_{1}$ and $p_{0} p_{2}$.

1.1. Drectional derivative $D f$ and the tangent cone. We denote by $K(\cdot)$ the Euclidean cone over a metric space (see $[\mathbf{1}, \S 4]$ for the definition of the Euclidean cone). The following fact is well-known:

Fact 1.1. The pointed Hausdorff $\operatorname{limit}_{\lim _{\varepsilon \rightarrow 0}}\left(\varepsilon^{-1} X, p\right)$ of the $\left(\varepsilon^{-1}\right)$-scaling of the metric around $p$ is isometric to the Euclidean cone $K\left(\Sigma_{p} X\right)$ for every $p \in X$.

We set $K_{p} X:=K\left(\Sigma_{p} X\right)$ and call it the tangent cone at $p$ in $X$. Let $p^{*}$ denote the vertex of $K_{p} X$ and $\alpha u$, for $\alpha \geq 0$ and $u \in \Sigma_{p} X$, the point in $K_{p} X$ such that $\left|\alpha u, p^{*}\right|=\alpha$ and $\operatorname{pr}(\alpha u)=u$, where $\operatorname{pr}: K_{p} X \backslash\left\{p^{*}\right\} \longrightarrow \Sigma_{p} X$ is the projection.

Let $f: X \longrightarrow R$ be a function of $D^{r}$ class and $1 \leq k \leq r$. Then we obtain the extension

$$
D f_{p, u_{1}, u_{2}, \ldots, u_{k-1}}: K_{u_{k-1}} K_{u_{k-2}} \cdots K_{u_{1}} K_{p} X \longrightarrow R
$$

of $d f_{p, u_{1}, u_{2}, \ldots, u_{k-1}}: \Sigma_{u_{k-1}} \Sigma_{u_{k-2}} \cdots \Sigma_{u_{1}} \Sigma_{p} X \longrightarrow R$ with the condition described as follows. We see that $K_{u_{k-1}} K_{u_{k-2}} \cdots K_{u_{1}} K_{p} X$ splits isometrically into the product

$$
K\left(\Sigma_{u_{k-1}} \Sigma_{u_{k-2}} \cdots \Sigma_{u_{1}} \Sigma_{p} X\right) \times\left\langle u_{1}, u_{2}, \ldots, u_{k-1}\right\rangle
$$

for every $\left(p, u_{1}, u_{2}, \ldots, u_{k-1}\right) \in \Sigma^{k-1} X$, where $\langle\cdot\rangle$ denotes the linear span. Under this identification, we have for $u=\left(\alpha_{k} u_{k}, \alpha_{k-1} u_{k-1}+\alpha_{k-2} u_{k-2}+\right.$ $\left.\cdots+\alpha_{1} u_{1}\right) \in K\left(\Sigma_{u_{k-1}} \Sigma_{u_{k-2}} \cdots \Sigma_{u_{1}} \Sigma_{p} X\right) \times\left\langle u_{1}, u_{2}, \ldots, u_{k-1}\right\rangle$

$$
D f_{p, u_{1}, u_{2}, \ldots, u_{k-1}}(u)=\sqrt{\alpha_{k}^{2} d f_{p, u_{1}, u_{2}, \ldots, u_{k-1}}\left(u_{k}\right)^{2}+\alpha_{k-1}^{2}+\cdots+\alpha_{1}^{2}} .
$$

In particular for $k=1$ in $(\dagger)$, we agree that $D f_{p}\left(\alpha_{1} u_{1}\right)=\alpha_{1} d f_{p}\left(u_{1}\right)$ for all $\alpha_{1} u_{1} \in K_{p} X$ and $u_{1} \in \Sigma_{p} X$. 
1.2. Generalized gradient and gradient curves. Let $f: X \longrightarrow R$ be a function of $D^{1}$ class. Then by the compactness of $\Sigma_{p} X, d f_{p}: \Sigma_{p} X \longrightarrow$ $R$ attains the maximum and minimum values on $\Sigma_{p} X$ for each $p \in X$. We denote by $M\left(d f_{p}\right)$ the maximum level set of $d f_{p}$ and by $m\left(d f_{p}\right)$ the minimum level set of $d f_{p}$. If $M\left(d f_{p}\right)$ consists of only one element, we express it by $\widehat{\nabla} f(p)$ and put $\nabla f(p):=|\nabla f|(p) \cdot \widehat{\nabla} f(p) \in K_{p} X$, where $|\nabla f|(p):=$ $\max _{u \in \Sigma_{p} X} d f_{p}(u)$. We call $\nabla f(p)$ the generalized gradient of $f$ at $p$. In this notation, if $m\left(d f_{p}\right)$ consists of only one element, $\widehat{\nabla}(-f)(p)$ coincides with the element of $m\left(d f_{p}\right)$.

A curve $c:[a, b] \longrightarrow X$ by definition has the right (left) tangent direction $v \in \Sigma_{c(t)}$ at $t \in[a, b)$ (resp. $t \in(a, b]$ ) if any initial direction of any minimal segment from $c(t+h)$ to $c(t)$ converges to $v$ as $h \downarrow 0$ (resp. $h \uparrow 0)$. The gradient curve $c:[a, b] \longrightarrow X$ of a function $g$ on $X$ is defined such that $c$ has the right tangent $\widehat{\nabla} g(c(t))$ for every $t \in[a, b)$.

Example 1.3. Let $\widetilde{X}$ be an Alexandrov space with curv $\geq-\kappa^{2}$. Then the metric product $X:=\widetilde{X} \times R$ is an Alexandrov space with curv $\geq-\kappa^{2}$. Define $\eta: X \longrightarrow R$ by $\eta((\widetilde{p}, t)):=t$. Then $\eta$ is a nontrivial affine function of $D^{2,2}$ class (see Proposition 3.2). Then $\Sigma_{p} X$ is the spherical suspension of $\Sigma_{p} \widetilde{X}$ with its suspension points $\nabla \eta(p)(=\widehat{\nabla} \eta(p))$ and $\nabla(-\eta)(p)(=\widehat{\nabla}(-\eta)(p))$ for every $p \in X$. If $\widetilde{X}$ has singular points, then so does $X=\widetilde{X} \times R$.

Example 1.4. Let $C$ be an unbounded convex body in $R^{n}$ with nonempty interior and with boundary. Then $C$ is a noncompact $n$-dimensional Alexandrov space with curv $\geq 0$ (with boundary). We take an arbitrary unit vector $z \in R^{n}$ and denote by $h_{z}: C \longrightarrow R$ the height function in the direction $z$, i.e., $h_{z}(p):=\langle z, p\rangle$, where $\langle$,$\rangle is the cannonical inner product in R^{n}$. Then $h_{z}$ is affine. If there is a point on the boundary of $C$ such that the diameter of $\Sigma_{p}$ is less than $\pi$, then $C$ does not split into the product of a line and a space, and then $h_{z}$ is of $D^{n}$ class but not of $D^{n, 1}$ class.

\section{Affine functions of $D^{1}$ class.}

Throughout this section we assume that $\varphi: X \longrightarrow R$ is an affine function of $D^{1}$ class. We first prove the following lemma, which will frequently be used in this paper.

Lemma 2.1. Fix an arbitrary point $p \in X$. Then $D \varphi_{p}: K_{p} X \longrightarrow R$ becomes an affine function again. In other words, we have

$$
(\sin |u, v|) d \varphi_{p}(\sigma(t))=\sin (|u, v|-t) d \varphi_{p}(u)+\sin t d \varphi_{p}(v)
$$

for all $u, v \in \Sigma_{p} X$, for every minimal geodesic $\sigma:[0,|u, v|] \longrightarrow \Sigma_{p} X$ from $u$ to $v$ and for every $t \in[0,|u, v|]$.

Differentiating $(*)$ in $t$ at $t=0$ yields the following: 
Corollary 2.2. We have the directional derivative of second order at $(p, u)$ $\in \Sigma X$

$(* *) \quad(\sin |u, v|) \tilde{d} \varphi_{p, u}(\dot{\sigma}(0))=d \varphi_{p}(v)-d \varphi_{p}(u) \cos |u, v|$,

where $\dot{\sigma}(0)$ is the initial direction of $\sigma$.

Proof of Lemma 2.1. From the continuity of $d \varphi_{p}$, it suffices to show $(*)$ for all $u, v \in \widetilde{\Sigma}_{p} X$ with $0<|u, v|<\pi$ and for $t \in(0,|u, v|)$. Identifying $u, v$ with two unit vectors in $R^{2}$ which makes angle $|u, v|_{\Sigma_{p} X}$, we define a number $\lambda=\lambda(t) \in(0,1)$ such that $\angle(u,(1-\lambda) u+\lambda v)=t$. Let $\gamma_{u}$ and $\gamma_{v}$ be the geodesics tangent to $u$ and $v$ respectively and $\left\{s_{i}\right\}$ a sequence of numbers such that $s_{i} \searrow 0$ as $i \rightarrow \infty$. We can choose an appropriate subsequence $\left\{s_{j}\right\} \subset\left\{s_{i}\right\}$ so that a sequence $\left\{\tau_{j}:[0,1] \longrightarrow\left(1 / s_{j}\right) X\right\}$ of minimal geodesics in $\left(1 / s_{j}\right) X$ from $\gamma_{u}\left(s_{j}\right)$ to $\gamma_{v}\left(s_{j}\right)$ tends to a segment $\tau$ on $K_{p} X$ as $j \rightarrow \infty$. Here each $\tau_{j}$ is parameterized proportionally to arclength, and the segment $\tau$ is projected to a minimal geodesic $\sigma:[0,|u, v|] \longrightarrow \Sigma_{p} X$ from $u$ to $v$.

Let $\alpha_{j}:\left[0,\left|p, \tau_{j}(\lambda)\right|\right] \longrightarrow X$ be a minimal geodesic from $p$ to $\tau_{j}(\lambda)$. By the continuity of $d \varphi_{p}$ and Fact 1.1, we have $d \varphi_{p}\left(\dot{\alpha}_{j}(0)\right) \rightarrow d \varphi_{p}(\sigma(t))$ as $j \rightarrow \infty$. Using Fact 1.1 and the definition of affine functions, we obtain

$$
d \varphi_{p}(\sigma(t))=\lim _{j \rightarrow \infty} d \varphi_{p}\left(\dot{\alpha}_{j}(0)\right)=\left[(1-\lambda) d \varphi_{p}(u)+\lambda d \varphi_{p}(v)\right] \frac{\sin t}{\lambda \sin |u, v|} .
$$

In elementary Euclidean geometry, we have $(1-\lambda) / \lambda=\sin (|u, v|-t) / \sin t$. Thus we obtain $(*)$.

For the first assertion, we need to prove that $d \varphi_{p}(u)=-d \varphi_{p}(v)$ for $u, v \in$ $\Sigma_{p} X$ with $|u, v|=\pi$. This is obvious from the continuity of $d \varphi_{p}$ and (*). Hence this completes the proof.

Lemma 2.3. If $\max _{u \in \Sigma_{p} X} d \varphi_{p}(u)>0\left(\min _{u \in \Sigma_{p} X} d \varphi_{p}(u)<0\right)$ at some point $p \in X$, then the maximum level set $M\left(d \varphi_{p}\right)$ of $d \varphi_{p}$ (resp. the minimum level set $m\left(d \varphi_{p}\right)$ of $\left.d \varphi_{p}\right)$ consists of only one element. In particular, the generalized gradient $\nabla \varphi(p)$ (resp. $\nabla(-\varphi)(p))$ is defined for all $p \in X$ with $\varphi(p)<\sup _{X} \varphi\left(\right.$ resp. $\left.\varphi(p)>\inf _{X} \varphi\right)$.

Proof. We prove this lemma only in the case $\max _{u \in \Sigma_{p} X} d \varphi_{p}(u)>0$. Suppose that $M\left(d \varphi_{p}\right)$ contains two elements $u_{1}$ and $u_{2}$ under the assumption $\max d \varphi_{p}>0$. If $\left|u_{1}, u_{2}\right|=\pi$, then we have $d \varphi_{p}\left(u_{1}\right)=-d \varphi_{p}\left(u_{2}\right)$ by Lemma 2.1. Hence $d \varphi_{p}\left(u_{1}\right)=\max d \varphi_{p}=0$, a contradiction to the assumption. Otherwise, if $\left|u_{1}, u_{2}\right| \neq \pi$, we have, for $t=\left|u_{1}, u_{2}\right| / 2$ in (*) along some minimal geodesic $\sigma:\left[0,\left|u_{1}, u_{2}\right|\right] \longrightarrow \Sigma_{p} X$ from $u_{1}$ to $u_{2}$, $d \varphi_{p}\left(u_{1}\right)=\max d \varphi_{p}<d \varphi_{p}\left(\sigma\left(\left|u_{1}, u_{2}\right| / 2\right)\right)$. This contradicts the choice of $u_{1}$. 
We discuss the zero level set $\left(d \varphi_{p}\right)^{-1}(0)$ of $d \varphi_{p}$. For simplicity, we put

$$
O\left(d \varphi_{p}\right):=\left(d \varphi_{p}\right)^{-1}(0) .
$$

If $o_{1}, o_{2} \in O\left(d \varphi_{p}\right)$ satisfy $0<\left|o_{1}, o_{2}\right|<\pi$, then Lemma 2.1 implies that all minimal geodesics from $o_{1}$ to $o_{2}$ are contained entirely in $O\left(d \varphi_{p}\right)$. Thus we obtain the following lemma:

Lemma 2.4. $O\left(d \varphi_{p}\right)$ is locally convex.

We deal with a point $p \in X$ such that $\varphi(p)<\sup _{X} \varphi$, or equivalently, $\max d \varphi_{p}>0$. Then by Lemma 2.3 , the generalized gradient $\nabla \varphi(p)$ is defined. For such point $p$, we investigate $\hat{\nabla} \varphi(p)$ and $O\left(d \varphi_{p}\right)$ in the following proposition:

Proposition 2.5. Let $p$ be a point of $X$ with $\varphi(p)<\sup _{X} \varphi$. Then the following (i) and (ii) hold:

(i) For every $o \in O\left(d \varphi_{p}\right)$, we have $|\widehat{\nabla} \varphi(p), o| \leq \pi / 2$.

(ii) If there are elements $o_{1}$ and $o_{2}$ of $O\left(d \varphi_{p}\right)$ such that $0<\left|o_{1}, o_{2}\right|<\pi$ and $\left|\widehat{\nabla} \varphi(p), o_{1}\right|=\left|\widehat{\nabla} \varphi(p), o_{2}\right|=\pi / 2$, then the triple $\left(\widehat{\nabla} \varphi(p), o_{1}, o_{2}\right)$ spans a totally geodesic triangular surface of constant curvature 1.

Remark 2.6. Since $-\varphi$ is also affine, the same assertions as above hold for $p \in X$ with $\varphi(p)>\inf _{X} \varphi$ and for $\widehat{\nabla}(-\varphi)(p)$ instead of $\widehat{\nabla} \varphi(p)$.

Proof of Proposition 2.5. (i) We want to use (**) in Corollary 2.2 for $u=\widehat{\nabla} \varphi(p)$ and $v=o \in O\left(d \varphi_{p}\right)$. If $|\widehat{\nabla} \varphi(p), o|=\pi$, then it follows from Lemma 2.1 that $\max _{u \in \Sigma_{p} X} d \varphi_{p}(u)=d \varphi_{p}(\widehat{\nabla} \varphi(p))=-d \varphi_{p}(o)=0$, a contradiction. Thus $|\widehat{\nabla} \varphi(p), o| \neq \pi$. Applying $(* *)$ to $\widehat{\nabla} \varphi(p)$ and $o$ along some minimal geodesic from $\widehat{\nabla} \varphi(p)$ to $o$ and using $\widetilde{d} \varphi_{p, \widehat{\nabla} \varphi(p)} \leq 0$, we have

$$
d \varphi_{p}(o)-|\nabla \varphi|(p) \cos |\widehat{\nabla} \varphi(p), o| \leq 0 .
$$

Hence $|\widehat{\nabla} \varphi(p), o| \leq \pi / 2$.

(ii) Draw a comparison triangle $\triangle\left(\overline{\widehat{\nabla} \varphi(p)}, \bar{o}_{1}, \bar{o}_{2}\right)$ in the unit sphere $S^{2}(1)$ corresponding to a geodesic triangle $\triangle\left(\widehat{\nabla} \varphi(p), o_{1}, o_{2}\right)$ in $\Sigma_{p} X$. Take a point $o_{3}$ in the interior of the edge $o_{1} O_{2}$ and a point $\bar{o}_{3}$ in the edge $\bar{o}_{1} \bar{o}_{2}$ corresponding to $o_{3}$. Then the Global Comparison Theorem implies that $\left|\widehat{\nabla} \varphi(p), o_{3}\right| \geq$ $\left|\widehat{\nabla} \varphi(p), \bar{o}_{3}\right|=\pi / 2$ (see Fact 1.0). Since $o_{3} \in O\left(d \varphi_{p}\right)$ by Lemma 2.4, it follows from (i) that $\left|\widehat{\nabla} \varphi(p), o_{3}\right| \leq \pi / 2$. Hence $\left|\widehat{\nabla} \varphi(p), o_{3}\right|=\left|\widehat{\widehat{\nabla} \varphi(p)}, \bar{o}_{3}\right|$. Therefore (ii) follows from the rigidity of geodesic triangle in the Global Comparison Theorem. 


\section{Affine functions of $D^{2,2}$ class.}

Proposition 3.1. If $\varphi: X \longrightarrow R$ is a nontrivial affine function of $D^{2,2}$ class, then the following (i)-(v) hold:

(i) $\Sigma_{p} X$ is the spherical suspension of $O\left(d \varphi_{p}\right)$ with its suspension points $\widehat{\nabla} \varphi(p)$ and $\widehat{\nabla}(-\varphi)(p)$ for every $p \in X$.

(ii) We have

$$
d \varphi_{p}(u)=|\nabla \varphi|(p) \cos |\widehat{\nabla} \varphi(p), u|_{\Sigma_{p} X}
$$

for every $p \in X$ and for every $u \in \Sigma_{p} X$, or equivalentlly,

$$
D \varphi_{p}(u)=|\nabla \varphi|(p)|u| \cos \angle_{p *}(\nabla \varphi(p), u)
$$

for every $u \in K_{p} X$. Here $\angle_{p *}$ denotes the angle distance at the vertex $p *$.

(iii) Fix two arbitrary numbers $a, b \in \varphi(X)$ with $a<b$. Then for every $q \in \varphi^{-1}(a)$ and for every minimal geodesic $\sigma_{q}:[0, l(q)] \longrightarrow X$ from $q$ to $\varphi^{-1}(b)$, we have

$$
\dot{\sigma}_{q}(0)=\widehat{\nabla} \varphi(q) \quad \text { and } \quad\left(\sigma_{q}^{-1}\right)(0)=\widehat{\nabla}(-\varphi)\left(\sigma_{q}^{-1}(0)\right),
$$

where $\sigma_{q}^{-1}:[0, l(q)] \longrightarrow X$ is defined by $\sigma_{q}^{-1}(t):=\sigma_{q}(l(q)-t), t \in$ $[0, l(q)]$.

(iv) There is a unique complete gradient curve $\phi: R \longrightarrow X$ of $\varphi$ passing through $p$ parameterized by $\varphi \circ \phi(t)=\varphi(p)+t, t \in R$, for every $p \in X$. Moreover, it satisfies

$$
\frac{\left|\varphi\left(\phi\left(t_{1}\right)\right)-\varphi\left(\phi\left(t_{2}\right)\right)\right|}{\left|\phi\left(t_{1}\right), \phi\left(t_{2}\right)\right|}=|\nabla \varphi|(\phi(t))=|\nabla \varphi|(p)
$$

for all $t, t_{1}, t_{2} \in R$, and in partucular $\phi$ is a straight line.

(v) $|\nabla \varphi|(p)$ is constant for all $p \in X$.

Proof. (i) Fix a point $p \in X$ arbitrarily. Since $\varphi$ is nontrivial, either $\max d \varphi_{p}>0$ or $\min d \varphi_{p}<0$ holds. If $\max d \varphi_{p}>0$, then $\min d \varphi_{p}<0$ since $\varphi$ is particularly of $D^{2,1}$ class. Similarly, we have $\max d \varphi_{p}>0$ if $\min d \varphi_{p}<0$. Thus $\widehat{\nabla} \varphi(p)$ and $\widehat{\nabla}(-\varphi)(p)$ at $p$ are defined.

By Proposition 2.5 (ii), it suffices to show the following:

Assertion. $|\widehat{\nabla} \varphi(p), o|_{\Sigma_{p} X}=|\widehat{\nabla}(-\varphi)(p), o|_{\Sigma_{p} X}=\pi / 2$ for all $o \in O\left(d \varphi_{p}\right)$.

Suppose that there is $o \in O\left(d \varphi_{p}\right)$ such that $|\widehat{\nabla} \varphi(p), o| \neq \pi / 2$. Then by Proposition 2.5 (i), $|\widehat{\nabla} \varphi(p), o|<\pi / 2$. Apply $(* *)$ to $\widehat{\nabla} \varphi(p)$ and $o$ along a minimal geodesic $\sigma:[0,|\widehat{\nabla} \varphi(p), o|] \longrightarrow \Sigma_{p} X$ from $\widehat{\nabla} \varphi(p)$ to $o$. Then we have $d \varphi_{p, \hat{\nabla} \varphi(p)}(\dot{\sigma}(0))<0$. Since $\varphi$ is particularly of $D^{2}$ class, there is a neighborhood $W$ of $\dot{\sigma}(0)$ in $\Sigma_{\widehat{\nabla} \varphi(p)} \Sigma_{p} X$ such that $d \varphi_{p, \widehat{\nabla} \varphi(p)}(w)<0$ for every 
$w \in \bar{W}$. Since $d \varphi_{p, \widehat{\nabla} \varphi(p)} \leq 0$ and $\bar{W}$ is of positive measure with respect to $(n-2)$-dimensional Hausdorff measure, we obtain

$$
\int_{\Sigma_{\widehat{\nabla} \varphi(p)} \Sigma_{p} X \ni w} d \varphi_{p, \widehat{\nabla} \varphi(p)}(w) d \mathcal{H}^{n-2}(w)<0 .
$$

This contradicts the assumption that $\varphi$ is of $D^{2,2}$ class.

(ii) It suffices to show the equation for $u \in \Sigma_{p} X \backslash\{\widehat{\nabla} \varphi(p), \widehat{\nabla}(-\varphi)(p)\}$. By (i), any such $u$ is contained in a minimal geodesic $\tau:[0, \pi] \longrightarrow \Sigma_{p} X$ joining two suspension points. Applying (**) to $\widehat{\nabla} \varphi(p)$ and $\tau(\pi / 2)$ along $\tau$, we have $d \varphi_{p, \widehat{\nabla} \varphi(p)}(\dot{\tau}(0))=0$. Using again $(* *)$ for $\widehat{\nabla} \varphi(p)$ and $u$ along $\tau$, we obtain $d \varphi_{p}(u)=|\nabla \varphi|(p) \cos |\widehat{\nabla} \varphi(p), u|$.

(iii) Suppose that $\dot{\sigma}_{q}(0) \neq \nabla \varphi(q)$ for some minimal geodesic $\sigma_{q}:[0, l(q)]$ $\longrightarrow X$ from $q$ to $\varphi^{-1}(b)$. Then we can find a broken geodesic

$$
\xi=\bigcup_{i} \gamma_{i}:[0, l(\xi)] \longrightarrow X
$$

such that $(\varphi \circ \xi)_{+}^{\prime}(s)>d \varphi_{q}\left(\dot{\sigma}_{q}(0)\right)$ for every $s \in[0, l(\xi))$ and $\xi(0)=q$, $\xi(l(\xi)) \in \varphi^{-1}(b)$. The construction of $\xi$ is achieved in the same way as in 2 -dimensional Alexandrov space (see [7, Lemma 2(2)]). Since $\varphi \circ \xi$ is almost everywhere differentiable, we conclude that $l(q)>l(\xi)$. This contradicts the minimizing property of $\sigma_{q}$.

(iv) Choose a double-ended sequence $\left\{a_{j}\right\}_{j \in Z}$ such that $a_{0}=\varphi(p), a_{j} \nearrow$ $\sup _{X} \varphi$ as $j \rightarrow \infty$ and $a_{j} \searrow \inf _{X} \varphi$ as $j \rightarrow-\infty$. We start from $p \in \varphi^{-1}\left(a_{0}\right)$ and repeat the same construction by minimal projections as in (iii). That is, let $p_{0}:=p$ and $p_{j+1}$ denote the foot of the (unique) minimal geodesic from $p_{j}$ to $\varphi^{-1}\left(a_{j+1}\right)$ for $j \geq 0$. For $j \leq 0$, let $p_{j-1}$ denote the foot of the (unique) minimal geodesic from $p_{j}$ to $\varphi^{-1}\left(a_{j-1}\right)$. Then we obtain the curve

$$
\phi:=\bigcup_{j \in Z} p_{j} p_{j+1}:\left(\inf _{X} \varphi-\varphi(p), \sup _{X} \varphi-\varphi(p)\right) \longrightarrow X
$$

parameterized by $\varphi \circ \phi(t)=\varphi(p)+t$. By the construction, we see that every subarc from $\phi\left(t_{1}\right)$ to $\phi\left(t_{2}\right)$ of $\phi$ is a minimal geodesic and

$$
\frac{\left|\varphi\left(\phi\left(t_{1}\right)\right)-\varphi\left(\phi\left(t_{2}\right)\right)\right|}{\left|\phi\left(t_{1}\right), \phi\left(t_{2}\right)\right|}=|\nabla \varphi|(\phi(t))=|\nabla \varphi|(p)
$$

for all $t, t_{1}, t_{2} \in\left(\inf _{X} \varphi-\varphi(p), \sup _{X} \varphi-\varphi(p)\right)$.

Once $\sup _{X} \varphi=\infty$ and $\inf _{X} \varphi=-\infty$ are established, the proof of (iv) is completed. Suppose that $\sup _{X} \varphi<\infty$. Then the sequence $\left\{p_{j}\right\}_{j=0,1, \ldots}$ accumulates to some point $p_{\infty}$. (i) implies that $\max d \varphi_{p_{\infty}}>0$ also at $p_{\infty}$. This is a contradiction to $\varphi\left(p_{\infty}\right)=\sup _{X} \varphi$. Therefore $\sup _{X} \varphi=\infty$. On the other hand, $\inf _{X} \varphi=-\infty$ follows from which $-\varphi$ is also affine. 
(v) Choose two points $p_{0}$ and $p_{1}$ arbitrarily. Let $\phi_{0}, \phi_{1}: R \longrightarrow X$ be two gradient curves passing through $p_{0}, p_{1}$ respectively obtained by (iv). We may assume from (iv) that $\varphi\left(p_{0}\right)=\varphi\left(p_{1}\right)$ and $\phi_{0} \neq \phi_{1}$. It is easily seen from (i) and (iv) that $\angle_{p_{1}}\left(p_{1} p_{0}, p_{1} \phi_{1}(t)\right)=\pi / 2,\left|p_{1}, \phi_{1}(t)\right|=t /|\nabla \varphi|\left(p_{1}\right)$ and $|\nabla \varphi|\left(p_{0}\right) \geq\left(\varphi\left(\phi_{1}(t)\right)-\varphi\left(p_{0}\right)\right) /\left|p_{0}, \phi_{1}(t)\right|=t /\left|p_{0}, \phi_{1}(t)\right|$ for all $t \in[0, \infty)$.

For every $t \in[0, \infty)$, draw a comparison hinge $\left(\bar{p}_{1} \bar{p}_{0}, \bar{p}_{1} \overline{\phi_{1}(t)}\right)$ of a hinge $\left(p_{1} p_{0}, p_{1} \phi_{1}(t)\right)$ in hyperbolic surface $H^{2}\left(-\kappa^{2}\right)$ of constant curvature $-\kappa^{2}$. Then the Global Comparison Theorem and the cosine formula in $H^{2}\left(-\kappa^{2}\right)$ implies that

$$
\left|p_{0}, \phi_{1}(t)\right| \leq\left|\bar{p}_{0}, \overline{\phi_{1}(t)}\right|=\frac{1}{\kappa} \cosh ^{-1}\left[\cosh \left(\kappa\left|p_{0}, p_{1}\right|\right) \cosh \left(\kappa\left|p_{1}, \phi_{1}(t)\right|\right)\right] .
$$

Therefore we have

$$
|\nabla \varphi|\left(p_{0}\right) \geq \frac{\kappa \cdot t}{\cosh ^{-1}\left[\cosh \left(\kappa\left|p_{0}, p_{1}\right|\right) \cosh \left(\kappa \cdot t /|\nabla \varphi|\left(p_{1}\right)\right)\right]} .
$$

Taking $t \rightarrow \infty$ and applying L'Hospital's formula, we obtain $|\nabla \varphi|\left(p_{0}\right) \geq$ $|\nabla \varphi|\left(p_{1}\right)$. The symmetric property of the above discussion implies the reverse inequality. This completes the proof.

We now assume that $\varphi: X \longrightarrow R$ is a nontrivial affine function of $D^{1}$ class satisfying the condition of the assertion (i) of Proposition 3.1. Then all other assertions (ii)-(v) follow. More precisely, the following holds:

Proposition 3.2. Let $\varphi: X \longrightarrow R$ be a nontrivial affine function of $D^{1}$ class. If $\varphi$ satisfies the condition that $\Sigma_{p} X$ forms the spherical suspension with its suspension points $\widehat{\nabla} \varphi(p)$ and $\widehat{\nabla}(-\varphi)(p)$ for every $p \in X$, then $\varphi$ is of $D^{n, n}$ class, $n=\operatorname{dim} X$.

Remark 3.3. It is easily seen that $\eta$ in Example 1.3 is of $D^{1}$ class and satisfies the assumption of Proposition 3.2. Hence $\eta$ is of $D^{2,2}$ class.

Proof of Proposition 3.2. We first prove that $\varphi$ is of $D^{1,1}$ class. Fix a point $p \in X$ arbitrarily. Since $\Sigma_{p} X$ is a spherical suspension with its suspension points $\widehat{\nabla} \varphi(p)$ and $\widehat{\nabla}(-\varphi)(p)$, there is a unique point $\underline{u} \in \Sigma_{p} X$ for every $u \in \Sigma_{p} X$ such that $|\widehat{\nabla} \varphi(p), u|=|\underline{u}, \widehat{\nabla}(-\varphi)(p)|$ and that $u, \underline{u}$ are lying on a common minimal geodesic joining suspension points. The correspondence $u \mapsto \underline{u}$ is a isometry between $d \varphi_{p}^{+}:=\left\{v \in \Sigma_{p} X \mid d \varphi_{p}(v) \geq 0\right\}$ and $d \varphi_{p}^{-}:=\left\{v \in \Sigma_{p} X \mid d \varphi_{p}(v) \leq 0\right\}$. Note that Proposition 3.1 (ii) is valid under the assumption of Proposition 3.2. Hence by Proposition 3.1 (ii), we have 
$d \varphi_{p}(\underline{u})=-d \varphi_{p}(u)$ for every $u \in \Sigma_{p} X$. A direct computation implies that

$$
\begin{aligned}
& \int_{\Sigma_{p} X \ni u} d \varphi_{p}(u) d \mathcal{H}^{n-1}(u) \\
& =\int_{d \varphi_{p}^{+} \ni u} d \varphi_{p}(u) d \mathcal{H}^{n-1}(u)+\int_{d \varphi_{p}^{-} \ni \underline{u}} d \varphi_{p}(\underline{u}) d \mathcal{H}^{n-1}(\underline{u})=0 .
\end{aligned}
$$

That is, $\varphi$ is of $D^{1,1}$ class.

We next show that $\varphi$ is of $D^{2,2}$ class. For every $w \in \Sigma_{\widehat{\nabla} \varphi(p)} \Sigma_{p} X$, there is a minimal geodesic $\sigma_{w}:[0, \pi] \longrightarrow \Sigma_{p} X$ from $\widehat{\nabla} \varphi(p)$ to $\widehat{\nabla}(-\varphi)(p)$ tangent to $w$. Apply $(* *)$ to $\hat{\nabla} \varphi(p)$ and $\sigma_{w}(\pi / 2)$ along $\sigma_{w}$. Then we have $d \varphi_{p, \widehat{\nabla} \varphi(p)}(w)=0$ for all $w \in \Sigma_{\widehat{\nabla} \varphi(p)} \Sigma_{p} X$. Therefore we conclude that $\varphi$ is of $D^{2,2}$ class at $(p, \widehat{\nabla} \varphi(p)) \in \Sigma X$ for all $p \in X$. Similarly, $\varphi$ is of $D^{2,2}$ class at $(p, \widehat{\nabla}(-\varphi)(p)) \in \Sigma X$ for all $p \in X$.

Therefore it suffices to show the $D^{2,2}$ condition for all $u \in \Sigma_{p} X \backslash\{\hat{\nabla} \varphi(p)$, $\widehat{\nabla}(-\varphi)(p)\}$. Such $u$ is contained in a minimal geodesic from $\hat{\nabla} \varphi(p)$ to $\widehat{\nabla}(-\varphi)(p)$. Letting $d_{\widehat{\nabla} \varphi}: \Sigma_{p} X \longrightarrow R$ denote the distance function from $\widehat{\nabla} \varphi(p)$, we see that $\Sigma_{u} \Sigma_{p} X$ is also a spherical suspension with its suspension points $\nabla\left(-d_{\widehat{\nabla} \varphi}\right)(u)$ and $\nabla d_{\widehat{\nabla} \varphi}(u)$. For every $w \in \widetilde{\Sigma}_{u} \Sigma_{p} X$ let $\sigma_{w}$ : $[0, l(w)] \longrightarrow \Sigma_{p} X$ be a geodesic tangent to $w$ and put $\theta_{w}:=\angle\left(\nabla\left(-d_{\widehat{\nabla} \varphi}(u), w\right)\right.$. Then by a direct computation, we have

$$
\widetilde{d} \varphi_{p, u}(w)=\left.\frac{d}{d t}\left(d \varphi_{p}\left(\sigma_{w}(t)\right)\right)\right|_{t=0}=|\nabla \varphi|(p) \sin |\widehat{\nabla} \varphi(p), u| \cos \theta_{w} .
$$

This means that $\widetilde{d} \varphi_{p, u}$ has the continuous extension $d \varphi_{p, u}: \Sigma_{u} \Sigma_{p} X \longrightarrow R$. Therefore $\varphi$ is of $D^{2,1}$ class. Moreover, the $D^{2,2}$ condition at $(p, u) \in \Sigma X$ is implied by the same computation as in the proof of the $D^{1,1}$ condition of $\varphi$.

Repeating the above computation, we see that $\varphi$ is of $D^{n, n}$ class.

\section{Totally geodesic flat strip spanned by gradient curves.}

In this section we prove Theorem A. Throughout this section let $\varphi: X \longrightarrow$ $R$ be a nontrivial affine function of $D^{2,2}$ class.

Let $p_{0}$ and $p_{1}$ be two arbitrary points with $\varphi\left(p_{0}\right)=\varphi\left(p_{1}\right)=: a$ and $\gamma:[0,1] \longrightarrow X$ a minimal geodesic from $p_{0}$ to $p_{1}$ parameterized to be proportional to arclength. By Proposition 3.1 (iv), there is a unique gradient curve $\phi_{\lambda}: R \longrightarrow X$ passing through $\gamma(\lambda)$ for every $\lambda \in[0,1]$.

We will prove in Proposition 4.3 that

$$
S:=\bigcup_{\lambda \in[0,1]} \phi_{\lambda}(R)
$$

is totally geodesic and flat. Once this is established, Theorem A easily follows. 
We first prove the following lemma:

Lemma 4.1. Fix $\lambda_{1}, \lambda_{2} \in[0,1]$ and $s_{0} \in R$ arbitrarily. Setting $l(s, t):=$ $\left|\phi_{\lambda_{1}}(s), \phi_{\lambda_{2}}(t)\right|$ for all $s, t \in R$, we have

$$
\lim _{h \rightarrow 0} \frac{l\left(s_{0}+h, s_{0}+h\right)-l\left(s_{0}, s_{0}\right)}{h}=0 .
$$

Proof. Let $\tau_{s t}:[0, l(s, t)] \longrightarrow X$ be a minimal geodesic from $\phi_{\lambda_{1}}(s)$ to $\phi_{\lambda_{2}}(t)$ and $\theta_{i}(s, t):=\angle\left(\tau_{s t}, \nabla \varphi\left(\phi_{\lambda_{i}}\right)\right), i=1,2$. We note that $\theta_{i}(s, t)$, $i=1,2$, is independent of the choice of the minimal geodesic $\tau_{s t}$. Actually, Proposition 3.1 (ii) implies that $|\nabla \varphi| \cos \theta_{1}(s, t)=d \varphi_{\phi_{\lambda_{1}}(s)}\left(\dot{\tau}_{s t}(0)\right)=$ $d \varphi_{\phi_{\lambda_{1}}(s)}\left(\dot{\tau}_{s t}^{\prime}(0)\right)=|\nabla \varphi| \cos \theta_{1}^{\prime}(s, t)$ for every other minimal geodesic $\tau_{s t}^{\prime}$ from $\phi_{\lambda_{1}}(s)$ to $\phi_{\lambda_{2}}(t)$ and for $\theta_{1}^{\prime}(s, t)=\angle\left(\tau_{s t}^{\prime}, \nabla \varphi\left(\phi_{\lambda_{1}}\right)\right)$. Similarly, we have $\theta_{2}^{\prime}(s, t)=\theta_{2}(s, t)$ for $\theta_{2}^{\prime}(s, t):=\angle\left(\tau_{s t}^{\prime}, \nabla \varphi\left(\phi_{\lambda_{2}}\right)\right)$. Therefore it follows from the first variation formula $([\mathbf{9}$, Theorem 3.5]) that for all $s, t \in R$, the partial derivatives $\frac{\partial l}{\partial s}(s, t)$ and $\frac{\partial l}{\partial t}(s, t)$ exist and equal $(-1 /|\nabla \varphi|) \cos \theta_{1}(s, t)$ and $(-1 /|\nabla \varphi|) \cos \theta_{2}(s, t)$ respectively. Thus we obtain for every $h \in R$,

$$
\left|l\left(s_{0}+h, s_{0}+h\right)-l\left(s_{0}+h, s_{0}\right)\right|=\frac{1}{|\nabla \varphi|}\left|\int_{0}^{|h|} \cos \theta_{2}\left(s_{0}+h, s_{0}+t\right) d t\right|
$$

and

$$
\left|l\left(s_{0}+h, s_{0}\right)-l\left(s_{0}, s_{0}\right)\right|=\frac{1}{|\nabla \varphi|}\left|\int_{0}^{|h|} \cos \theta_{1}\left(s_{0}+t, s_{0}\right) d t\right| .
$$

Proposition 3.1 implies that for every $\varepsilon>0$ there is $\delta=\delta(\varepsilon)>0$ such that $\left|\theta_{1}(s, t)-\pi / 2\right|,\left|\theta_{2}(s, t)-\pi / 2\right| \leq \varepsilon$ for all $s, t \in\left[s_{0}-\delta, s_{0}+\delta\right]$. Therefore we have for all $h \in[-\delta, \delta]$,

$$
\left|l\left(s_{0}+h, s_{0}+h\right)-l\left(s_{0}, s_{0}\right)\right| \leq \frac{2}{|\nabla \varphi|}|h|\left|\cos \left(\frac{\pi}{2} \pm \varepsilon\right)\right| .
$$

Since $\delta(\varepsilon) \rightarrow 0$ as $\varepsilon \rightarrow 0$, we obtain the desired equality.

Define $c_{s}:[0,1] \longrightarrow S$ by $c_{s}(\lambda):=\phi_{\lambda}(s)$ for an arbitrarily fixed $s \in R$. Then the following holds:

Corollary 4.2. The curve $c_{s}$ is minimal for every $s \in R$.

Proof. Let $\operatorname{pr}_{a}: X \longrightarrow \varphi^{-1}(a)$ be the minimal projection to $\varphi^{-1}(a)$ and $\tau$ a minimal geodesic from $\phi_{0}(s)$ to $\phi_{1}(s)$. Then Lemma 4.1 implies that

$$
L\left(c_{s}\right) \geq L(\tau)=L\left(p r_{a} \circ \tau\right) \geq L(\gamma)=L\left(p r_{a} \circ c_{s}\right)=L\left(c_{s}\right),
$$

where $L(\cdot)$ means the length of a curve. This completes the proof.

The proof of Theorem A is completed by the following:

Proposition 4.3. The strip $S$ is totally geodesic and flat. 
Proof. Let $y_{0}, y_{1}$ be two points in $S$ and $\tau:[0,1] \longrightarrow X$ a minimal geodesic from $y_{0}$ to $y_{1}$ parameterized proportionally to arclength. We may assume that $y_{0} \in \phi_{0}(R)$ and $y_{1} \in \phi_{1}(R)$. Moreover, we may assume from Corollary 4.2 that $\varphi\left(y_{0}\right)<\varphi\left(y_{1}\right)$. We define a curve $c:[0,1] \longrightarrow S$ in $S$ by $c(\lambda):=\phi_{\lambda}\left((1-\lambda) \varphi\left(y_{0}\right)+\lambda \varphi\left(y_{1}\right)\right)$. Then $\varphi \circ c$ is an affine function. We will calculate the length of $c$. We denote by $p r_{\phi_{\lambda}}: X \longrightarrow \phi_{\lambda}(R)$ the minimal projection to $\phi_{\lambda}(R)$ and put $y_{1}^{\prime}:=p r_{\phi_{1}}\left(y_{0}\right)$. Fix $\lambda \in(0,1)$ arbitrarily and let $h \in R \backslash\{0\}$ be a number such that $|h|$ is sufficiently small. We consider the triangle $\triangle\left(c(\lambda), c(\lambda+h), p r_{\phi_{\lambda+h}}(c(\lambda))\right)$ whose sides are all minimal if $h>0$ (if $h<0$ consider $\triangle\left(c(\lambda), c(\lambda+h), p r_{\phi_{\lambda}}(c(\lambda+h))\right)$ ). This is a right triangle on $X$. By Lemma 4.1 and the parameterization of $c$, we have for $h>0$,

$$
\left|c(\lambda), p r_{\phi_{\lambda+h}}(c(\lambda))\right|=L(\gamma)|h| \quad \text { and } \quad\left|p r_{\phi_{\lambda+h}}(c(\lambda)), c(\lambda+h)\right|=\left|y_{1}, y_{1}^{\prime}\right||h|,
$$

and for $h<0,\left|c(\lambda), p r_{\phi_{\lambda}}(c(\lambda+h))\right|=L(\gamma)|h|$ and $\mid p r_{\phi_{\lambda}}(c(\lambda+h)), c(\lambda+$ $h)|=| y_{1}, y_{1}^{\prime}|| h \mid$. Note that $c(\lambda)$ is a point of the straight line $\phi_{\lambda}$. Fact 1.0 together with this imply that $|c(\lambda), c(\lambda+h)|=\sqrt{L(\gamma)^{2}|h|^{2}+\left|y_{1}, y_{1}^{\prime}\right|^{2}|h|^{2}}+$ $o(|h|)$. Equivalently,

$$
|\dot{c}|(\lambda):=\lim _{h \rightarrow 0} \frac{|c(\lambda), c(\lambda+h)|}{|h|}=\sqrt{L(\gamma)^{2}+\left|y_{1}, y_{1}^{\prime}\right|^{2}}
$$

for all $\lambda \in(0,1)$. This implies that $c$ is a Lipschitz curve. Therefore $L(c)$ is calculated as

$$
L(c)=\int_{0}^{1}|\dot{c}|(\lambda) d \lambda=\sqrt{L(\gamma)^{2}+\left|y_{1}, y_{1}^{\prime}\right|^{2}} .
$$

A similar calculation shows that $L\left(p r_{a} \circ \tau\right)=\sqrt{L(\tau)^{2}-\left|y_{1}, y_{1}^{\prime}\right|^{2}}$, where $p r_{a}$ is the same as in the proof of Corollary 4.2. Thus we obtain

$$
L(c)^{2} \geq L(\tau)^{2}=L\left(p r_{a} \circ \tau\right)^{2}+\left|y_{1}, y_{1}^{\prime}\right|^{2} \geq L(\gamma)^{2}+\left|y_{1}, y_{1}^{\prime}\right|^{2}=L(c)^{2} .
$$

This completes the proof.

Acknowledgments. The author would like to express his thanks to Y. Otsu, K. Shiohama, T. Shioya and T. Yamaguchi for their valuable advice. The notion of functions of $D^{r, s}$ class is introduced by Y. Otsu.

\section{References}

[1] Yu.D. Burago, M. Gromov and G. Perelman, A.D. Alexandrov's spaces with curvatures bounded below I, Russian Math. Surveys, 47(2) (1992), 1-58, MR 93m:53035.

[2] J. Cheeger and D. Gromoll, The splitting theorem for manifolds of nonnegative Ricci curvature, J. Diff. Geom., 61 (1971), 119-128, MR 46 \#2597, Zbl 0223.53033. 
[3] R.E. Greene and K. Shiohama, The isometry groups of manifolds admitting nonconstant convex functions, J. Math. Soc. Japan, 39(1) (1987), 1-16, MR 88a:53032, Zbl 0611.53039.

[4] K. Grove and S. Markvorsen, New extremal problems for the Riemannian recognition program via Alexandrov geometry, J. Amer. Math. Soc., 8 (1995), 1-28, MR 95j:53066, Zbl 0829.53033.

[5] K. Grove and F. Wilhelm, Hard and soft packing radius theorems, Ann. of Math., 142(2) (1995), 213-237, MR 96h:53054, Zbl 0846.53042.

[6] N. Innami, Splitting theorems of Riemannian manifolds, Compositio Math., 47 (1982), 237-247, MR 84e:53053, Zbl 0514.53040.

[7] Y. Mashiko, Affine functions on Alexandrov surfaces, Osaka J. Math., 36 (1999), 853-859, MR 2001a:53057.

[8] A. Milka, Metric structure of one class of spaces containing straight lines (in Russian), Ukrain. Geom. Sbornik, 4 (1967), 43-48, MR 41 \#983.

[9] Y. Otsu and T. Shioya, The Riemannian structure of Alexandrov spaces, J. Diff. Geom., 39 (1994), 629-658, MR 95e:53062, Zbl 0808.53061.

[10] G. Perelman and A. Petrunin, Quasigeodesics and gradient curves in Alexandrov spaces, preprint.

[11] T. Shioya, Splitting theorems for nonnegatively curved open manifolds with large ideal boundary, Math. Z., 212 (1993), 223-238, MR 93k:53036, Zbl 0791.53045.

[12] V.A. Toponogov, Spaces with straight lines, AMS Transl., 37 (1964), 278-280, MR 21 \#7520, Zbl 0138.42902.

Received October 17, 2000.

Faculty of Science And EngineERING

SAgA UNIVERSITY

HOJYOUMACHI 1

SAGA 840-8502, JAPAN

E-mail address: mashiko@ms.saga-u.ac.jp 